\title{
Approaches to establishing voltage control requirements for distributed generation units
}

\author{
Pavel Ilyushin ${ }^{1 *}$, Petr Berezovskiy ${ }^{2}$, and Sergey Filippov ${ }^{3}$ \\ ${ }^{1}$ Petersburg power engineering institute of professional development, Aviation str., 23, Saint-Petersburg, Russia \\ ${ }^{2}$ Study committee C6 of CIGRE Russian national committee, Aviation str., 23, Saint-Petersburg, Russia \\ ${ }^{3}$ Energy research institute of the Russian academy of sciences, Nagornaya str., 31, bld. 2, Moscow, Russia
}

\begin{abstract}
Analysis of Russian national regulation and technical standards requirements for generating equipment participation in voltage and reactive power control in the power system carried out. Summary of some international experience in voltage control organization under conditions of distributed generation units' deep integration in public grid is presented in article. Advantages of voltage and reactive power control automation over decentralized and poorly coordinated control are substantiated. Problematic aspects of distributed generation units (including RES) integration, as well as ENTSO-E approaches to voltage and reactive power control in the synchronous zone of continental Europe are considered. Technical requirements of Deutsch and Danish regulations, regarding participation of distributed generation facilities in voltage control are given. Approaches to establishing voltage control requirements for distributed generation units' operation with national power system are presented.
\end{abstract}

\section{Introduction}

The Russian business community certainly seems interested in distributed generation (DG), as businesses seek cheaper energy, while DG is an effective tool to produce it. Another reason to use DG in industry is that some critical consumers need uninterrupted power supply, as the technology they use tolerates no blackout.

In Russia and worldwide, wind farms and solar farms are on the rise. As a result of contest-based selection of renewable-energy projects, Russia is expected to construct 5,278.3 MW of solar and wind generation until $2024[1]$.

All the generator sets (GS) at DG facilities can generate reactive power, thus affecting the reactive power flow across grids as well as the voltage level at load nodes. Given how many DG facilities Russia has, it is crucial to refer to international experience of using DG for voltage and reactive power flow control to draft sufficient Russia-specific technical requirements (TR) to the GS designed for operating in parallel with the grid.

\section{International voltage control experience}

Connecting the generator sets at DG facilities, including renewable source-based ones, to the grid adds a significant number of new generators to the grid. This complicates voltage and reactive power flow control while also continually raising the dimensionality of the control problem. On the other hand, grid reliability and electricity quality requirements are ever higher on the consumer bus side.

In this context, decentralized barely coordinated grid control based on «manual» adjustment of automatic excitation controls (AEC) in electric machines, switching the static capacitor batteries (SCB) and power transformer under voltage regulation (UVR) by visual assessment of a limited set of local parameters does not seem efficient [2].

Coordinated control over all reactive power sources is necessary to maximize the positive effect, which can only be done by computing the steady states (SS) for each grid section in particular.

In some countries of Europe, secondary voltage and reactive power flow controls are based on splitting the grid into control zones on the basis of maximum reciprocal electrical remoteness. Each control zone has control plants connected to high voltage (HV) and ultrahigh voltage (UHV) grids; such plants have greatest reactive power control range and maximum impact on the voltage in the local controlled grids. Zonal voltage controllers (ZVC) will automatically maintain a set voltage at the reference points (RP) of their control zones by reconfiguring the AEC thresholds in the controlling power units.

Each control plant has local plant-wide voltage control systems (PVCS), which maintain a set voltage on the plant buses and help ensure the non-ambiguous distribution of reactive power between the power units by affecting the AEC configurations.

As far as possible, ZVC provide independent control for their reference points and enable unambiguous

* Corresponding author: ilyushin.pv@mail.ru 
distribution of reactive power between control stations (plants) within each zone by adjusting the PVCS input setpoints. Thus, ZVC coupled with PVCS provide secondary voltage control for RP and control-plant buses. Besides, ZVC are also tasked to switch the SCP; to control the shunting reactors (SR), the transformer UVR, and the static reactive power compensators so as to release some range of control at zonal control power plants. In case a control zone only has one control plant, ZVC functionality is handled by the PVCS of this plant. This voltage and reactive power control structure for a single control zone is nearly identical to the Russiandesigned automatic nodal voltage controller (ANVC) made to control concentrated generating nodes (CGN) [3].

Tertiary voltage control (TVC) is being studied as an alternative that will provide safer and more efficient grid-wide controls. TVC is expected to minimize the deviation in actual voltage readings from the optima for control points other than control-zone reference points. By controlling the adherence to the specified voltage curves, the TVC will trigger voltage adjustments; both tertiary and secondary controls operate in a closed circuit.

It is the targeted allocation of more or less independent control zones plus coordinating the performance of the above-mentioned control circuits that stabilizes the tri-circuit automatic voltage control system comprising the generator $\mathrm{AEC}$, the $\mathrm{ZVC}$, and the TVC. Thus, the generator AEC time constant is about 0.3 to $0.5 \mathrm{~s}$; the time constant of secondary control circuits provided by PVCS must be about 3 to $5 \mathrm{~s}$; the secondary control time constant for plant buses and control zone reference points must be about 30 to $50 \mathrm{~s}$. TVC must affect ZVC at 5 to 20 minutes. The input lag between different levels of the control hierarchy should not exceed $2 \mathrm{~s}$. The untied performance of different control circuits enables them to function separately and nearly independently while the transient response remain aperiodic [4].

Primary reactive power and voltage control is the task of AEC used in generators and synchronous compensators (SC), as well one of the automatic voltage controllers (AVC) controlled by substation-based reactive power compensators (RPC). Within the set reactive power control range, the AEC of synchronous machines will keep the output voltage readings within 2$4 \%$ of the design value. They operate continuously in any grid mode and are adjusted to the grid transfer functions and control algorithms.

\section{ENTSO-E transition to requirements regulation in the synchronous zone of continental Europe}

The pan-European system code, ENTSO-E, regulates the matter of connecting any generating node to the grid; as such, it has been approved as consistent with the Regulations of the European Commission [5]. The document does not specify the requirements to the synchronous zone of continental Europe in terms of allocating a reactive power range for generator sets with an installed capacity of up to $50 \mathrm{MW}$ (type $B$ ); instead, national operators can specify such requirements for themselves. However, for type $B$ synchronous GS, AEC is mandatory by law; reactive current must be injected in case of voltage dips caused by multiphase SC (the rule is mandatory for wind farms).

\subsection{German experience}

In Germany, DG facilities mostly use renewable sourcebased GS (both wind farms and solar farms), which are in most cases connected to the distribution grid. The historical vertically-oriented structure of the energy system is slowly but steadily switching to distributed generation as renewable energy evolves.

The basic regulatory documents that govern the DG GS involvement in voltage control are [6] for the GS in low-voltage (LV) grids and [7] for their medium-voltage counterparts. The TR [6] are mainly focused on getting and keeping the solar farms up and running. Such farms are mainly used in Southern Germany. The TR [7] are mainly intended for wind farms common in Northern Germany, as well as for sundry generation types.

For low-voltage energy systems and grids, the code mainly seeks to provide technical requirements to voltage maintenance and reactive power generation at solar farms; another area of focus is to reduce such facilities' active power generation shall the grid-wide frequency rise.

One of the most problematic parts of integrating DG facilities is to raise the voltage at DG nodes [8]. If more active power is generated in an LV grid than could consumed, whereby the resistance exceeds the reactance, the GS connection point has its voltage increased by $>10 \%$ of the rated value $(230 \mathrm{~V})$. In case of excessively long feeders, the LV transformer substations must keep their low voltage above the rated values so that the consumer connection points always have a voltage of $207 \mathrm{~V}$ (minimum).

When using a conventional approach to voltage control in LV grids, solar farms connected to respective feeders trigger ever more actions involving LV/MVtransformer UVR, making them more expensive to maintain and repair [9]. Figures $1 a$ and $1 b$ clarify the above-mentioned problems of solar farms in a LV grid.

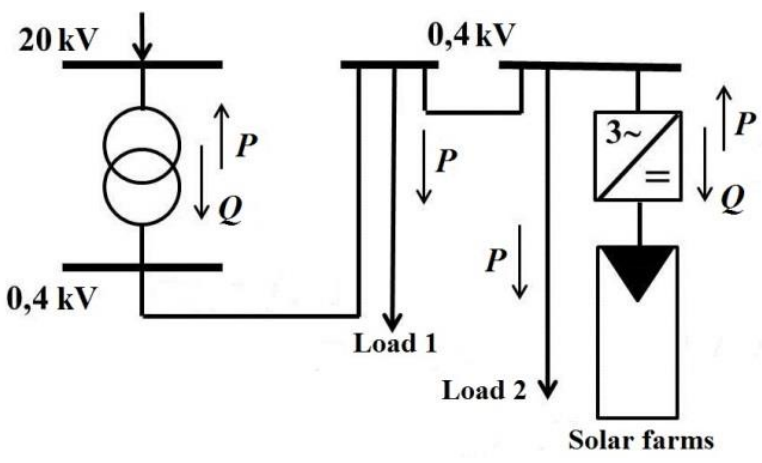

a) 


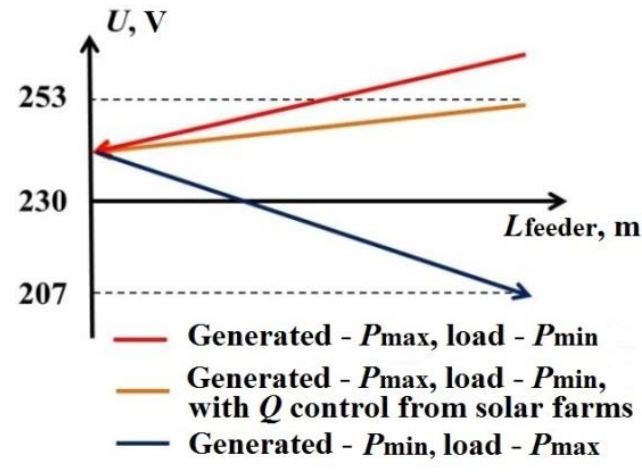

b)

Fig. 1. Solar-farms problems: (a) simplified single-line distribution grid circuit; (b) $0.4-\mathrm{kV}$ grid curves in different operating situations.

The Code [5] presents requirements to keeping the GS up and running in a range from $\cos \varphi=0.95$ (underexcitation) to $\cos \varphi=0.95$ in case of overexcitation, whereby the active power generation must equal at least $20 \%$ of the rated value, see Figure $2 a$. In case the total installed capacity of a GS located at a common location point exceeds $13.8 \mathrm{kVA}$, the control range is from $\cos \varphi=0.9$ for underexcitation to $\cos \varphi=0.9$ for overexcitation, see Figure $2 b$.

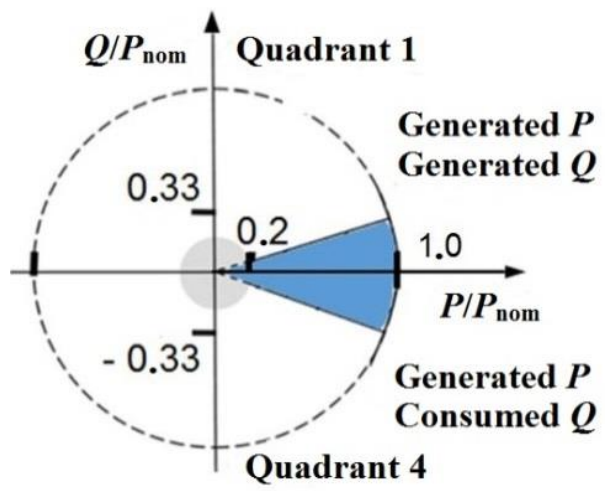

a)

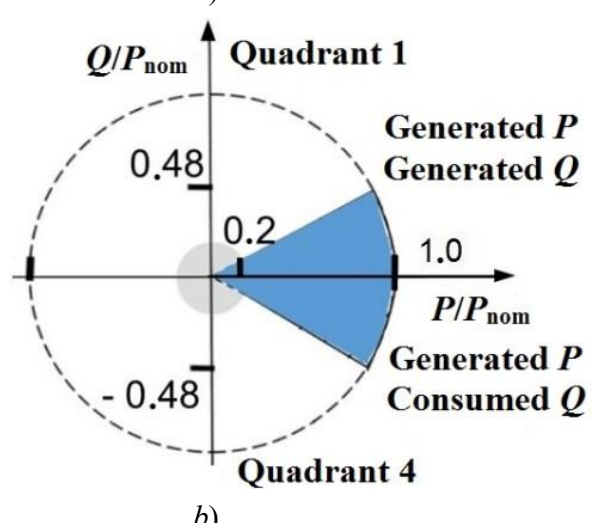

b)

Fig. 2. Requirements to GS in case of under- and overexcitation within the power factor range: (a) $\cos \varphi=0.95$; (b) $\cos \varphi=0.9$.

The GS reactive power must adjust automatically to the changes in active power in compliance with $\cos \varphi$. The method for finding the required performance by reactive power $(\cos \varphi=$ const or active power-affected $\cos \varphi$ ) shall be chosen by the National operator. The GS must reach the reactive power suitable for set performance within 10 seconds. This does not apply to direct-switch GS. Figure 3 presents typical reactive power control performance.

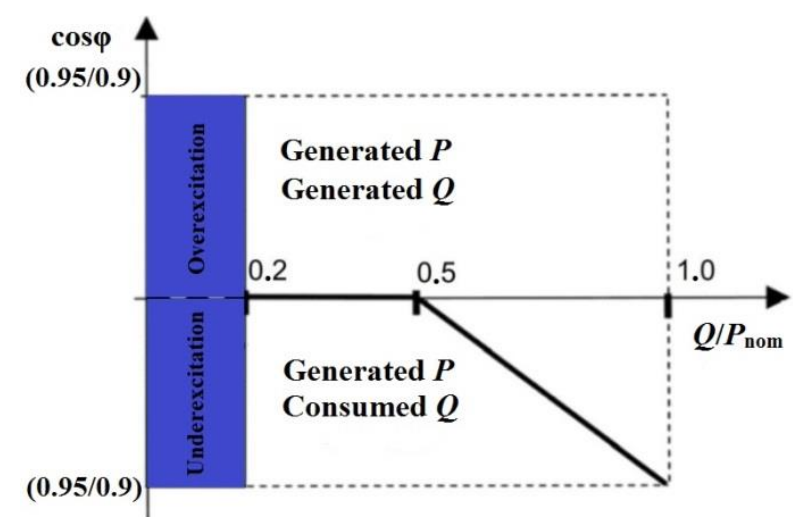

Fig. 3. Typical reactive power control performance.

Document [7] stipulates with respect to medium voltage (MV) grids and networks that the GS must be able to keep the facility running within a $\cos \varphi$ range from 0.95 (underexcitation) and 0.95 (overexcitation). For GS running at 0 to $10 \%$ of rated power, the reactive power output or consumption must not exceed $10 \%$ of the maximum coordinated active power, while reducing the active power to control the GS connection point is not subject to penalties unlike Iceland's configuration.

\subsection{Danish experience}

Unlike most foreign energy systems, the Danish grids have spent over two decades to build distributed infrastructure with large-scape DG implementation. Active integration of renewable source-based GS did not affect the energy development system; instead, it replacing one set of generation technologies with another set.

Electricity-related regulatory acts are developed by Energinet.dk, the sole regulator of Denmark's electricity transmission and gas transport infra-structure. Energinet.dk requirements to connecting to an energy grid are a set of five documents:

- Requirements to generation facilities up to $11 \mathrm{~kW}$ in power, type-agnostic [10],

- Requirements to solar farms $>11 \mathrm{~kW} \mathrm{[11],}$

- Requirements to combined heat and power plant $(\mathrm{CHPP})>11 \mathrm{~kW}[12]$,

- Requirements to wind farms $>11 \mathrm{~kW} \mathrm{[13],}$

- Requirements to energy storage systems [14].

Consider requirements to solar farms and wind farms. Any such farm is expected to be able to control at least one of its parameters: reactive power output at the grid connection point (regardless of the active power generation figures); $\cos \varphi$ (reactive power as a function of the active power output); or the control point voltage, which is affected after the FASs has received a specific command within 10 seconds. 
Solar farms are also requirement to be able to automatically alter $\cos \varphi$ as soon as the control point reaches a certain threshold, or reactive power output does. Usually, the said function is triggered as soon as the control point voltage reaches $105 \%$ of the rated value; $\cos \varphi$ is configured as follows:

$$
\begin{aligned}
& 1-\left(P / P_{\text {nom }}=0.0 ; \cos \varphi=1.00\right) ; \\
& 2-\left(P / P_{\text {nom }}=0,5 ; \cos \varphi=1.00\right) ; \\
& 3-\left(P / P_{\text {nom }}=1.0 ; \cos \varphi=0.90\right) .
\end{aligned}
$$

At an equal installed power, the reactive power adjustment range is limited at $\cos \varphi=0.90$ for solar farms and $\cos \varphi=0.995$ for wind farms (50 kW to $1.5 \mathrm{MW}$ ) or $\cos \varphi=0.975(1.5 \mathrm{MW}$ to $25 \mathrm{MW})$. Denmark has somewhat stricter requirements to reactive power control at solar farms.

Wind farms of the above-mentioned power class are not facing the control point voltage control requirements (only for wind farms $>25 \mathrm{MW}$ ), neither are they subject to automatic $\cos \varphi$ requirements. The specified requirement to solar farms only applies to low-power facilities (50 kW to $1.5 \mathrm{MW})$, which means this function is mainly intended to handle voltage control in low- and medium-voltage grids with numerous renewable sourcebased GS within non-dispatchable households.

\section{Russian technical requirements to DG GS}

Given the specifics of Russian 0.4-kV distribution grids, it is advisable to formulate the Russian technical requirements to DG GS with respect to their involvement in grid-wide voltage.

It is also advisable to set a lower limit of microgeneration facility power, to which voltage control TR will not apply at $1 \mathrm{~kW}$. Technical requirements to DG facilities ranging from $1 \mathrm{~kW}$ to $0.5-1 \mathrm{MW}$ connected to $0.4-\mathrm{kV}$ grids should be structured as follows:

- Requirements to generation facilities 1 to $15 \mathrm{~kW}$ in power, type-agnostic, MW,

- Requirements to solar farms, $15 \mathrm{~kW}$ to $0.5-1$

- Requirements to wind farms, $15 \mathrm{~kW}$ to $0.5-1$ MW,

- Requirements to CHPP, $15 \mathrm{~kW}$ to $0.5-1 \mathrm{MW}$.

It is advisable to set technical requirements to DG facilities connected to medium-voltage $6-$ to $35-\mathrm{kV}$ grids, $0.5-1 \mathrm{MW}$ to $5 \mathrm{MW}$ in power, as well as to medium-voltage DG facilities, 5 to $25 \mathrm{MW}$ in power, with a similar gradation.

Note that when connecting a $>5$ MW DG facility, the applicant must design the power output circuitry and have it approved by the grid operator as well as by the energy dispatcher.

For setting technical requirements to DG facilities, it is necessary to consider both the DG GS and the operating features of distribution grids where such facilities are to be used. Here are some of them:

- GS excitation systems based on gas-turbine and gas-piston engines, equipped with automatic excitation controls that usually use four control algorithms. As such, the algorithm selection is subject to regulation,

- Lowering the grid voltage will drastically drop the maximum available GS reactive power, imposing a heavier load on the grid when operating in forced, repair, or post-emergency mode, whence lower voltage,

- GS involvement in secondary voltage control requires taking into account the maximum available GS reactive power and how it could be raised by partial active-power offload,

- Readjustment of AEC voltage setpoints at a single DG facility will trigger an opposite readjustment of reactive power at other DG facilities, which requires coordinated GS AEC readjustments,

- Using gas-turbine and gas-piston GS for secondary voltage control to reduce voltage spikes requires taking into account the constraints on minimum reactive power output, which is necessary for the prevention of static destabilization and overheating of stator ends,

- Using a load harmonization module as part of the GS AEC might trigger a voltage collapse at industrialload nodes, which means its usability must be tested by calculating the electromechanical transients.

In the light of international TR standardization experience, the first regulatory document drafts might contain only the minimum necessary requirements to be further refined and amended with better and more extensive operational and project experience as more and more DG facilities are commissioned and can be studied in terms of their effects on the grid.

Thus, the TR to DG facilities must be developed in packages with due account for the Russian grid features, the distribution grid voltage and reactive power flow control principles, as well as DG GS features.

\section{Conclusion}

Integrating the DG generator units, including wind and solar farms, does increase the number of generators operating in the grid, which complicates the problem of voltage control and reactive power flow controls in the grid while also making this approach a high-dimensional one.

Barely coordinated grid control based on "manual" adjustment of AEC in electric machines, switching the static capacitor batteries (SCB) and power transformer UVR by visual assessment of a limited set of local parameters does not seem efficient.

A three-circuit automatic voltage control system can function reliably by targeted allocation of more or less independent control zones and coordinating the control circuit performance for their nearly independent functioning, in which case the transients in each circuit remain aperiodic.

The international experience of drafting the regulations on DG GS is mostly that of seeking to stabilize the operation of grids using numerous renewable sources for generation, as well as to maintain the high quality of electricity in low- and mediumvoltage grids. 
Europe's experience shows that the TR to allocating a reactive power range and to the involvement in voltage control are part of the mandatory requirements to DG GS for integration in grids; such TR are part of the European commission's regulations and can be elaborated at the national level.

Russian requirements to DG GS for their involvement in voltage control must be based on the international experience; however, they must also take into account the specifics of Russian grids and use breakdowns by voltage, GS type and power.

\section{References}

1. P. Ilyushin, A. Pazderin, R. Seit, Proceedings 17th International Ural Conference on AC Electric Drives, ACED (2018).

2. S. Makarovsky, Z. Khvoshchinskaya, Energetik, 6, 18-21 (2002).

3. L. Rosman, Means and control systems in the energy sector, Overview information, Issue 2 (1987).

4. P. Gorozhankin, A. Mayorov, S. Makarovsky, A. Rubtsov, Power Plant, 6, 40-47 (2008).

5. Commission Regulation (EU) 2016/631 establishing a network code on requirements for grid connection of generators (2016).

6. VDE-AR-N 4105: 2018-11. Power generation systems connected to the low-voltage distribution network (2018).

7. BDEW, Guideline for generating plants' connection to and parallel operation with the medium-voltage network (2008).

8. Y. Wu, S. Tsai, M. Zou, Energy and Power Engineering, 5, 209-214 (2013).

9. Y. Agalgaonkar, B. Pal, R. Jabr, IEEE Transactions on Power Systems, 29(1), 182-192 (2014).

10. Energinet.dk, Technical regulation 3.2.1 for power plants up to and including $11 \mathrm{~kW}(2016)$.

11. Energinet.dk, Technical regulation 3.2 .2 for $P V$ power plants above $11 \mathrm{~kW}$ (2016).

12. Energinet.dk, Technical regulation 3.2.3 for thermal plants above $11 \mathrm{~kW}$ (2017).

13. Energinet.dk, Technical regulation 3.2.5 for wind power plants above $11 \mathrm{~kW}(2016)$.

14. Energinet.dk, Technical regulation 3.3.1 for battery plants (2017). 